PROCEEDINGS OF THE

AMERICAN MATHEMATICAL SOCIETY

Volume 130, Number 10, Pages 3097-3099

S 0002-9939(02)06390-6

Article electronically published on April 17, 2002

\title{
A COUNTEREXAMPLE CONCERNING WHITNEY REVERSIBLE PROPERTIES
}

\author{
JANUSZ J. CHARATONIK AND WŁODZIMIERZ J. CHARATONIK
}

(Communicated by Alan Dow)

\begin{abstract}
Two properties of continua are shown which are strong Whitney reversible while not sequential strong Whitney reversible. This answers an old question of S. B. Nadler, Jr.
\end{abstract}

A continuum means a compact, connected metric space, and a mapping means a continuous transformation. By an $n$-od (for an integer $n>2$ ) we understand a continuum $X$ containing a subcontinuum $Z$ such that $X \backslash Z$ has at least $n$ components. Given a continuum $X$ with a metric $d$, we denote by $C(X)$ the hyperspace of all subcontinua of $X$ equipped with the Hausdorff metric (see e.g. 2, (0.1), p. 1 and (0.12), p. 10]). A Whitney map for $C(X)$ is a mapping

$$
\mu: C(X) \rightarrow[0, \infty)
$$

such that:

(0.1) $\mu(A)<\mu(B)$ for every two $A, B \in C(X)$ such that $A \subset B$ and $A \neq B$;

(0.2) $\mu(A)=0$ if and only if $A \in F_{1}(X)$.

For the concept and existence of a Whitney map see [1, Section 13, pp. 105-110]. For each $t \in[0, \mu(X)]$ the preimage $\mu^{-1}(t)$ is called a Whitney level. It is known that each Whitney level is a continuum; see $[1$, p. 159].

Let $\mathcal{P}$ be a topological property. We write $X \in \mathcal{P}$ to denote that a space $X$ has the property $\mathcal{P}$. A property $\mathcal{P}$ is said to be:

- a strong Whitney-reversible property (briefly $(s W r p)$ ) provided that for each continuum $X$ the following implication holds:

(0.3) if there is a Whitney map $\mu: C(X) \rightarrow[0, \infty)$ such that for all $t \in(0, \mu(X))$ we have $\mu^{-1}(t) \in \mathcal{P}$, then $X \in \mathcal{P}$;

- a sequential strong Whitney-reversible property (briefly (ssWrp)) provided that for each continuum $X$ the following implication holds:

$(0.4)$ if there are a Whitney map $\mu: C(X) \rightarrow[0, \infty)$ and a sequence $\left\{t_{n}: n \in \mathbb{N}\right\}$ in $(0, \mu(X))$ such that $\lim t_{n}=0$ and for each $n \in \mathbb{N}$ we have $\mu^{-1}\left(t_{n}\right) \in \mathcal{P}$, then $X \in \mathcal{P}$.

The definitions are taken from [3] compare 11. Definitions 27.1 (c) and (d), pp. 232 and 233].

Received by the editors March 27, 2001 and, in revised form, May 9, 2001.

2000 Mathematics Subject Classification. Primary 54B20, 54E40, 54F15.

Key words and phrases. Continuum, hyperspace, Whitney map, Whitney property.

(C)2002 American Mathematical Society 
S. B. Nadler, Jr. asks in [2, Remark and question 14.55.1, p. 463] if there is an (sWrp) which is not an (ssWrp). The same question is repeated 21 years later in [1, Question 27.2, p. 233]. In this note we answer the question in the affirmative.

Let $M_{3}$ be the union of a 2-cell $D$ and of three mutually disjoint $\operatorname{arcs} A_{1}, A_{2}, A_{3}$ such that $A_{i} \cap D$ is an end point of $A_{i}$ for each $i \in\{1,2,3\}$ (see [1. Figure 56 (b), p. 431] for a picture). Further, let $\mathcal{P}_{1}$ be the property "to be homeomorphic to $M_{3}$ ". We start with the following proposition.

Proposition 1. There is no continuum $X$ such that there exists a Whitney map $\mu: C(X) \rightarrow[0, \infty)$ all positive Whitney levels of which have property $\mathcal{P}_{1}$.

Proof. Suppose on the contary that there are a continuum $X$ and a Whitney map $\mu: C(X) \rightarrow[0, \infty)$ such that for each $t \in(0, \mu(X))$ the condition $\mu^{-1}(t) \in \mathcal{P}_{1}$ holds. Note that local connectedness is an $(s W r p),[1,52.2$, p. 281], and that some Whitney levels of a 4-od contain 3-cubes, [2, (14.33), p. 430]. Thus:

(1.1) $X$ is locally connected;

(1.2) $X$ does not contain any 4-od.

It follows from (1.1) and (1.2) that the continuum $X$ is

(1.3) either an arc, or a simple closed curve, or a simple triod, or a noose.

(A noose is the union of a simple closed curve and an arc whose intersection is one of the end points of the arc; see [1, p. 36]). For none of these four continua in (1.3) do all Whitney levels have property $\mathcal{P}_{1}$; in particular, large Whitney levels of a simple triod are 2-cells [1, Theorem 65.10 (c), p. 309]. The proof is complete.

Theorem 2. Property $\mathcal{P}_{1}$ is an $(s W r p)$ and it is not an (ssWrp).

Proof. It follows from Proposition 1 that $\mathcal{P}_{1}$ satisfies the definition of an $(s W r p)$ (in an empty way, since no continuum having the needed property exists). To see that $\mathcal{P}_{1} \notin(s s W r p)$ note that if $T$ is a simple triod, and $\mu: C(T) \rightarrow[0, \infty)$ is a Whitney map, then the Whitney levels $\mu^{-1}(t)$ for small positive numbers $t$ have property $\mathcal{P}_{1}$, [1. Example 65.4, pp. 307-308], while $T \notin \mathcal{P}_{1}$.

As it is said in the beginning of the proof of Theorem 2, property $\mathcal{P}_{1}$ is an $(s W r p)$ in an empty way. Since this can be seen as defect of our solution of the Nadler problem mentioned above, we will consider another property, $\mathcal{P}_{2}$, for which this imperfection is eliminated.

Let $\mathcal{P}_{2}$ be the property "to be homeomorphic either to an arc or to $M_{3}$ ".

Theorem 3. Property $\mathcal{P}_{2}$ is an $(s W r p)$ and it is not an $(s s W r p)$.

Proof. As in the proof of Theorem 2, a simple triod $T$ shows that $\mathcal{P}_{2} \notin(s s W r p)$. Further, arguing as previously in the proof of Proposition 1, we see that of the four continua in (1.3) only the arc has all Whitney levels with $\mathcal{P}_{2}$. Since obviously $\mathcal{P}_{2}$ holds for an arc, it follows that $\mathcal{P}_{2} \in(s W r p)$. The proof is complete.

Remark 4. The reader can consider other continua to answer Nadler's question: for example the continuum $M_{n}$ instead of $M_{3}$, for $n>3$ (see [1, Figure 56 (a), p. 431]) and/or a simple closed curve in place of an $\operatorname{arc}$ for $\mathcal{P}_{2}$. 


\section{REFERENCES}

[1] A. Illanes and S. B. Nadler, Jr., Hyperspaces, M. Dekker, New York and Basel, 1999. MR 99m:54006

[2] S. B. Nadler, Jr., Hyperspaces of sets, M. Dekker, New York and Basel, 1978. MR 58:18330

[3] S. B. Nadler, Jr., Whitney-reversible properties, Fund. Math. 109 (1980), 235-248. MR 82b:54044

Mathematical Institute, University of WrocŁaW Pl. Grunwaldzki 2/4, 50-384 WrocŁaw, Poland - and - Instituto de Matemáticas, UnAM Circuito Exterior, CiuDAd Universitaria, 04510 MÉxico, D. F., MÉxico

E-mail address: jjc@math.unam.mx

Mathematical Institute, University of WrocŁaw Pl. Grunwaldzki 2/4, 50-384 WrocŁaw, Poland - and - Department of Mathematics and Statistics, University of Missouri-Rolla, Rolla, Missouri 65409-0020

E-mail address: wjcharat@umr.edu 